\title{
CO MAPPING OF BARRED SPIRAL GALAXIES
}

\author{
N. KUNO, N. NAKAI, K. NISHIYAMA AND K. SORAI \\ Nobeyama Radio Observatory \\ Minamimaki-mura, Minamisaku-gun, Nagano 384-13 \\ T. HANDA \\ Inst. of Astronomy, Univ. of Tokyo \\ Osawa, Mitaka, Tokyo 181
}

AND
T. IGA
Shinsyu Univ.
Asahi, Matsumoto, Nagano 390

\section{Introduction}

We have made CO mapping observations of nearby barred spiral galaxies (NGC253, NGC3504, NGC4303, NGC4321, NGC6951, M83) with the Nobeyama 45-m telescope using the 2X2 SIS focal-plane array receiver. The purpose of this project is to investigate distribution and kinematics of molecular gas in barred spiral galaxies and the relation between star formation and them. We present some of the results (NGC3504 and NGC6951).

\section{NGC3504}

Figure 1(a) shows the integrated intensity map of $\mathrm{CO}$ emission of NGC 3504. The map shows that the molecular gas distributes along the leading edges of the optical bar. Since the position angles of the line of node and the semimajor axis of the bar of the galaxy are almost the same (Kenney et al. 1993), the velocity along the line of sight can be regarded as the velocity component perpendicular to the bar. A position-velocity diagram perpendicular to the bar shows that the velocity decreases at the molecular ridge abruptly. It means that the molecular gas moves along the ridge when it enters the ridge as shown by numerical simulations (e.g., Athanassoula 1992). The amplitude of the shift is about $50 \mathrm{~km} \mathrm{~s}^{-1}$ in the $\mathrm{P}-\mathrm{V}$ diagram and about $130 \mathrm{~km} \mathrm{~s}^{-1}$ in the galactic plane assuming an inclination angle 

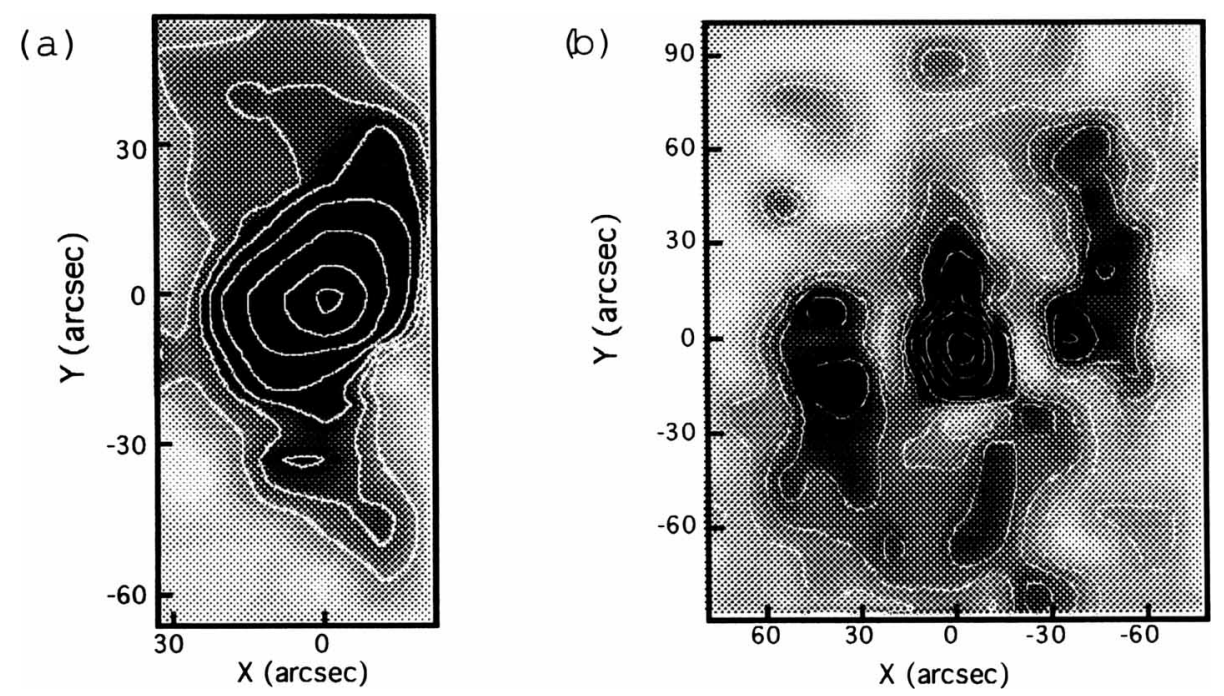

Figure 1. Integrated intensity map of CO emission of (a) NGC3504 and (b) NGC6951. $\mathrm{X}$ and $\mathrm{Y}$ are parallel to the minor and major axes of each galaxy, respectively.

of $22^{\circ}$. It is larger than the velocity shift found in the spiral arms in other galaxies. For example, even in M51 which has a strong density wave, the shift is $70-90 \mathrm{~km} \mathrm{~s}^{-1}$ across the arms (Kuno et al. 1997).

\section{NGC6951}

Figure 1(b) shows the integrated intensity map of CO emission of NGC 6951. In addition to the central peak, the $\mathrm{CO}$ emission is concentrated on the end of the bar and the spiral arms, while it depletes in the bar region. The large-scale distribution of the molecular gas coincides with the $\mathrm{H} \alpha$ image (Marquez and Moles 1993). The structure elongated from the center toward the north can be seen in the CO map. The radial distribution of the surface density of the molecular gas shows a secondary peak at the radius of the end of the bar. Such secondary peak is often seen in barred spiral galaxies (Nakai 1992). All galaxies of our sample have the same trend except for NGC3504 which is the earliest type galaxy of our sample.

\section{References}

Athanassoula, E. 1992, MNRAS 259, 345

Kenney, J.D.P., Carlstrom, J.E., Young, J.S. 1993, ApJ 418, 687

Kuno, N., Nakai, N. 1997, PASJ 49, 279

Marquez, I., Moles, M. 1993, AJ 105, 2090

Nakai, N. 1992, PASJ 44, L27 